\title{
O tipo de polimento altera a força explosiva de membros inferiores em atletas de voleibol?
}

http://dx.doi.org/10.11606/1807-5509201900010135

\author{
Leonardo de Sousa FORTES* \\ Hugo Augusto Alvares da Silva LIRA* \\ Geraldo José Santos OLIVEIRA* \\ Jeferson Macedo VIANNA** \\ Tony Meireles dos SANTOS*

\section{Resumo}

0 objetivo foi analisar o efeito do tipo de polimento na força explosiva de membros inferiores (FEMI) em jovens atletas de voleibol. Participaram 42 atletas com idade entre 15 e 17 anos, divididos aleatoriamente em 3 grupos: linear (GL), por etapa (GE) e controle (GC). Todos os grupos fizeram a mesma planificação de treinamento até a fase do polimento. Somente o GC não realizou polimento. 0 polimento teve duração de 3 semanas, adotando-se o método de polimento linear para o GL e polimento por etapa para o GE. Utilizou-se a plataforma de força para avaliar a FEMI. Este instrumento foi utilizado antes do início da temporada e na última semana de cada mesociclo. Conduziu-se a análise univariada de covariância (ANCOVA) de medidas repetidas para comparar a FEMI entre os grupos em função do mesociclo. Os resultados apresentaram efeitos de tempo $\left(F_{(4,38)}=31,93, p=0,01\right)$ e grupo $\left(F_{(3,39)}=41,87, p=0,01\right)$. A FEMI aumentou no $\operatorname{GL}\left(F_{(2,13)}=32,55\right.$, $p=0,01)$ e $\mathrm{GE}\left(F_{(2,12)}=29,14, p=0,01\right)$ após o polimento, fato não revelado para o $\mathrm{GC}\left(F_{(2,11)}=3,48, p=0,19\right)$. De maneira geral, os achados do presente estudo revelaram o aumento da FEMI após o polimento para GL e GE. Concluiu-se que o polimento foi eficaz para melhorar a FEMI de atletas de voleibol.

Palavras-chave: Força; Desempenho; Atletas.

\section{Introdução}

O voleibol é um esporte coletivo, caracterizado por açóes de alta intensidade e curta duração, intercaladas por momentos de baixa intensidade ${ }^{1,2}$. Atletas bem sucedidos no voleibol geralmente demonstram superioridade em alguns indicadores de desempenho, a saber: tomada de decisão, conhecimento tático processual, velocidade/agilidade e força explosiva de membros inferiores ${ }^{2}$.

Força explosiva, por sua vez, refere-se à capacidade do músculo de desenvolver altíssimos gradientes de força em pouquíssimo tempo ${ }^{3}$. A maximização da força explosiva depende das estratégias de treinamento físico e da recuperação fisiológica proporcionada aos atletas ${ }^{4}$.

De maneira geral, atletas de voleibol são submetidos a rotinas diárias de treinamento físico, técnico, tático e mental com a premissa de otimizar o desempenho esportivo. Neste sentido, aconselha-se que os treinadores de voleibol manipulem as cargas, meios e métodos de treino em períodos específicos com objetivos bem definidos ${ }^{4}$. A divisão dos objetivos do treinamento em fases consecutivas com a premissa de acarretar adaptaçóes morfofuncionais positivas é denominada periodização do treinamento esportivo ${ }^{5}$.

As fases consecutivas do treinamento, também conhecidas como mesociclos ${ }^{6}$, diferem na forma de prescriçáo dos parâmetros de treinamento (volume, intensidade, descanso e tipo de exercício). A periodização linear adota o aumento progressivo da intensidade e concomitante atenuação do volume de treinamento ${ }^{5}$, acarretando maximizaçáo do desempenho dos atletas ao final deste processo $^{6}$. Na periodização linear o treinador 
busca manipular as cargas de treinamento com o intuito de que seus atletas atinjam o melhor desempenho em uma única competição ao final do macrociclo 5 . Este tipo de periodização é composta por três etapas, a saber: preparatório, polimento e competitivo. A primeira fase é utilizada para se aumentar progressivamente volume e intensidade do treinamento ${ }^{5}$. Neste período é aumentado a frequência de realização de sessóes de alta intensidade ao mesmo tempo que o volume de treinamento é reduzido e/ou mantido 5 .

O polimento, por sua vez, é considerado uma fase da periodizaçáo que visa reduzir a fadiga imposta pelo treinamento sem que o atleta perca as adaptaçóes psicofisiológicas ${ }^{7}$. Assim, o polimento é utilizado para se reduzir o volume de todos os componentes das sessóes de treino, embora a intensidade seja mantida ${ }^{8}$. De acordo com MujıKA' ${ }^{9}$ o polimento deve ter duração de duas a quatro semanas. Estudos revelam que, ao final da fase de polimento, o tamanho das fibras musculares é aumentado, a velocidade do recrutamento de fibras musculares é otimizada e o complexo PGC-1a é ativado, o que, de certo modo, pode explicar a melhora da força explosiva e velocidade em atletas ${ }^{6,8}$.

Existem dois tipos de polimento: linear/progressivo e por etapa ${ }^{9}$. O primeiro diz respeito a diminuição sistemática e linear da carga de treino, ao passo que o polimento por etapa se refere a atenuaçáo repentina da carga de treino em uma quantidade constante. De acordo com Bosquet et al. ${ }^{8}$, existem dois métodos de polimento, pois atletas podem responder de forma diferente as cargas de treinamento. Logo, embora o polimento linear/progressivo possa ser mais eficaz para aumentar o rendimento em alguns atletas, o polimento por etapa pode ser mais efetivo para outros. Todavia, do melhor de nosso conhecimento, não existe investigação que tenha buscado analisar o efeito de diferentes tipos de polimento no desempenho de atletas de esporte coletivo.

Estudos têm indicado aumento do desempenho de atletas após a etapa do polimento ${ }^{10,11}$, embora tais investigaçóes tenham sido conduzidas com atletas de esportes cíclicos. Vale salientar que ainda não há consenso na literatura científica concernente a qual estratégia de polimento é mais eficaz para maximizar o desempenho de atletas de esportes intermitentes. Talvez isso possa ser explicado em razão da maior parte das modalidades esportivas de característica intermitente ser coletiva. Tratandose de esportes coletivos, o calendário não permite que as equipes alcancem um pico de desempenho, considerando a duraçáo e congestionamento de jogos ao longo de toda a temporada. Destacase, ainda, que a prescriçáo do polimento parece não ser unanimidade para muitos treinadores ${ }^{12}$. Treinadores creem que nas semanas antecedentes a competição-alvo tanto volume quanto intensidade devem ser aumentados ${ }^{11}$.

Do ponto de vista prático, este tipo de investigação poderá apontar o efeito de diferentes tipos de polimento sobre a força explosiva de membros inferiores, considerada um indicador de desempenho essencial para os atletas de voleibol. No entanto, embora o calendário competitivo do voleibol de alto nível normalmente seja muito extenso, vale salientar que existem torneios menores para equipes amadoras que apresentam duraçáo de 1 a 2 semanas. Neste sentido, investigar o efeito da fase do polimento na força explosiva de jovens atletas de voleibol torna-se importante para treinadores de equipes que disputem eventos competitivos com curta duração. Logo, os achados poderão ser de extrema importância para os treinadores de equipes amadoras desta modalidade esportiva. Em razáo dos apontamentos citados acima, o objetivo da investigaçáo foi analisar o efeito do tipo de polimento sobre a força explosiva de membros inferiores em jovens atletas de voleibol.

Por conseguinte, algumas hipóteses foram formuladas em virtude dos achados de duas revisóes sistemáticas ${ }^{6,8}$. Assim, considerando que os dois métodos de polimento (linear e por etapa) geram alteraçóes neurais positivas (tamanho das fibras musculares e a velocidade do recrutamento de fibras musculares), construiu-se a seguinte hipótese: a) as duas estratégias de polimento geram melhora na força explosiva de membros inferiores em atletas de voleibol. Por fim, considerando que a reduçáo repentina do volume de treinamento (adotado no polimento por etapa) pode não ser efetiva para o polimento com duração de mais de duas semanas ${ }^{6,8}$, formulou-se a segunda hipótese da investigação: b) o polimento linear maximiza mais a força explosiva de membros inferiores em atletas de voleibol do que o polimento por etapa. 


\section{Método}

\section{Participantes}

Trata-se de uma investigação experimental com duraçáo de 10 semanas desenvolvida com jovens atletas de voleibol do sexo masculino. A análise de dimensão amostral foi conduzida no software G*Power 3.1. Adotou-se poder de 0,80, $\alpha=0,05$ e tamanho do efeito de 0,50 . Assim, foi revelado o tamanho amostral de 39 sujeitos como necessário para se conduzir o experimento. Este tipo de análise foi realizado com a premissa de se evitar erro do tipo II e determinar o número mínimo de participantes para a investigação.

A amostra foi selecionada de forma não probabilística, sendo composta por 49 voluntários com idade entre 15 e 17 anos, participantes do campeonato pernambucano de voleibol das categorias sub-15 $(\mathrm{n}=22)$ e sub$17(\mathrm{n}=27)$. Os participantes foram divididos aleatoriamente de forma estratificada em três grupos: periodização linear $(\mathrm{GL}, \mathrm{n}=16)$, periodização por etapa (GE, $n=17)$ e grupo controle $(G C, n=16)$. Salientase que não se identificaram diferenças estatísticas para idade $\left(F_{(3,46)}=2,91, p=0,25\right)$, percentual de gordura $\left(F_{(3,46)}=2,44, p=0,22\right)$ e força explosiva de membros inferiores $\left(F_{(3,46)}=1,99, p=0,18\right)$ entre os grupos antes do início da investigação.

Os atletas treinavam em média $2 \mathrm{~h}$ por dia, com frequência de cinco vezes por semana. Para serem incluídos na pesquisa, os atletas deveriam: a) ser atleta de voleibol a pelo menos dois anos; b) treinar sistematicamente voleibol por pelo menos 8 h por semana; e c) estar inscrito no Campeonato Estadual de Voleibol, organizado pela Federação Pernambucana de Voleibol.

Contudo, 7 atletas foram excluídos em razão de faltarem mais do que 5\% das sessóes de treinamento no decorrer da investigação (10 semanas). Logo, a investigação contou com uma amostra final de 42 atletas $(\mathrm{GL}=15, \mathrm{GE}=14 \mathrm{e} \mathrm{GC}=13)$.

Após receber informação sobre os procedimentos aos quais seriam submetidos, os participantes assinaram um termo de assentimento. Os responsáveis dos atletas assinaram o termo de consentimento livre e esclarecido (TCLE), concordando com os procedimentos metodológicos da investigaçáo. Os procedimentos adotados neste estudo atenderam às normas $\mathrm{da}$ Resolução 466/12 do Conselho Nacional de Saúde para pesquisas em seres humanos. O projeto obteve aprovação do Comitê de Ética e Pesquisa em Seres Humanos da Universidade Federal de Pernambuco (CAE - 46978515.6.0000.5208).

\section{Desenho experimental}

Todos os grupos fizeram a mesma planificação de treinamento até as últimas três semanas antes do início da fase competitiva (TABELA 1). O GL realizou polimento linear, ao passo que o GE utilizou o método de polimento por etapa, conforme recomendações de Bosquet et al. ${ }^{8}$. Somente o GC náo realizou polimento. $\mathrm{O}$ polimento teve duraçáo de 3 semanas. Para o GL, foi reduzido somente o volume de treinamento: $80 \%$ para a primeira semana, $60 \%$ para a segunda e $40 \%$ na terceira semana (FIGURA 1), seguindo indicaçóes de Mujıka, Chaouachi e Chamari ${ }^{7}$. Para o GE, o volume de treinamento foi atenuado para $50 \%$ no decorrer das 3 semanas de polimento (FIGURA 2), de acordo com os apontamentos de Mujika, Chaouachi e Chamari ${ }^{7}$. O GC prolongou o mesociclo "preparatório II" até a data do início do campeonato estadual de voleibol (FIGURA 3).

A carga interna de treinamento foi quantificada adotando a média diária do método da percepção subjetiva do esforço da sessão (PSE-sessão) ${ }^{1,2}$. Após 30 minutos do fim de cada sessão de treinamento os atletas responderam a seguinte questão: "Como foi o seu treino?". Solicitou-se ao atleta que demonstrasse a percepçáo de intensidade da sessão a partir da escala de Borg de 10 pontos $(0=$ repouso a $10=$ máximo esforço $)$, conforme o método desenvolvido por Foster et al. ${ }^{13}$. Calculou-se o produto dos valores demonstrados pela escala da PSE e a duração em minutos de cada sessão, expressando, assim, a carga interna da sessão de treinamento. A carga semanal de treino foi calculada após cada microciclo. A carga semanal de treino foi obtida a partir da soma das cargas diárias e dividida por cinco (número de sessóes semanais de treino). A carga de cada mesociclo (preparatório I, preparatório II e polimento) foi calculada a partir da média das respectivas cargas semanais, conforme metodologia adotada em outra pesquisa ${ }^{2}$. Ressalta-se que os atletas foram familiarizados com o método PSE-sessão por um período de 10 dias antes do início da investigação. A carga interna de cada mesociclo do GL, GE e GC pode ser visualizada nas FIGURAS 1, 2 e 3, respectivamente.

O teste de impulsão vertical foi realizado pelos atletas antes do início da temporada, o que foi denominado como pre-teste, e na última semana de cada mesociclo [Preparatório I, Preparatório II e Polimento (somente para GL e GE)]. 
GL: periodização linear; GE: periodização por etapa; Pre: Preparatório; Pol: Polimento; Fis: Treinamento Físico; TÉC: treinamento técnico; TÁT: treinamento tático;

Tp: treinamento com pesos; $\mathrm{Ca}$ : circuito aeróbio; Tac: treinamento aeróbio contínuo; Ag: agilidade; Pot: potência anaeróbia alática; $\mathrm{Ra}$ : resistência anaeróbia; Pa: passe; Bloc: bloqueio; Saq: Saque; Td: treinamento do sistema defensivo; To: treinamento do sistema ofensivo.

GL: periodização linear. Cl: Carga interna de treinamento (mesociclo); U.A.: unidades arbitrárias; a $p<0,05$ em relação ao período preparatório I; $b p<0,05$ em relação ao período preparatório Il; $c p<$ 0,05 em relação ao polimento.

GE: periodização por etapa; $\mathrm{Cl}$ : Carga interna de treinamento (mesociclo); U.A.: unidades arbitrárias; $\mathrm{a} p<0,05$ em relação ao período preparatório I; ${ }^{b} p<0,05$ em relação ao período preparatório II; ${ }^{c}$ $p<0,05$ em relação ao polimento.
TABELA 1 - Descrição da frequência dos tipos de treinamento dos jovens atletas de voleibol em razão do mesociclo (preparatório I, preparatório II e polimento) e grupo (GL, GE e GC)
GL
GE
GC

\begin{tabular}{|c|c|c|c|c|c|c|c|c|c|}
\hline Meso & Pre I & Pre II & Pol & Pre I & Pre II & Pol & Pre I & Pre II & Pre II \\
\hline \multicolumn{10}{|l|}{ FÍS } \\
\hline $\mathrm{Tp}$ & 6 & 8 & 3 & 6 & 8 & 3 & 6 & 8 & 8 \\
\hline $\mathrm{Ca}$ & 4 & 6 & 2 & 4 & 6 & 2 & 4 & 6 & 6 \\
\hline $\mathrm{Tac}$ & 5 & 6 & 2 & 5 & 6 & 2 & 5 & 6 & 6 \\
\hline $\mathrm{Ag}$ & 3 & 5 & 3 & 3 & 5 & 3 & 3 & 5 & 5 \\
\hline Pot & 4 & 7 & 4 & 4 & 7 & 4 & 4 & 7 & 7 \\
\hline $\mathrm{Ra}$ & 3 & 4 & 3 & 3 & 4 & 3 & 3 & 4 & 4 \\
\hline $\mathrm{Ag}$ & 3 & 4 & 3 & 3 & 4 & 3 & 3 & 4 & 4 \\
\hline \multicolumn{10}{|l|}{ TÉC } \\
\hline $\mathrm{Pa}$ & 6 & 8 & 4 & 6 & 8 & 4 & 6 & 8 & 8 \\
\hline Bloc & 5 & 7 & 4 & 5 & 7 & 4 & 5 & 7 & 7 \\
\hline Saq & 8 & 8 & 5 & 8 & 8 & 5 & 8 & 8 & 8 \\
\hline \multicolumn{10}{|l|}{ TÁT } \\
\hline $\mathrm{Td}$ & 4 & 7 & 5 & 4 & 7 & 5 & 4 & 7 & 7 \\
\hline To & 3 & 6 & 5 & 3 & 6 & 5 & 3 & 6 & 6 \\
\hline
\end{tabular}

FIGURA 1 - Periodização do treinamento para o GL.

\begin{tabular}{|c|c|c|c|c|c|c|c|c|c|c|}
\hline \multirow{2}{*}{$\begin{array}{l}\text { Mesociclo } \\
\text { Microciclo }\end{array}$} & \multicolumn{3}{|c|}{ Preparatório I } & \multicolumn{4}{|c|}{ Preparatório II } & \multicolumn{3}{|c|}{ Polimento } \\
\hline & 1 & 2 & 3 & 4 & 5 & 6 & 7 & 8 & 9 & 10 \\
\hline \multicolumn{11}{|l|}{ Volume (\%) } \\
\hline \multicolumn{11}{|l|}{100} \\
\hline \multicolumn{11}{|l|}{90} \\
\hline \multicolumn{11}{|l|}{80} \\
\hline \multicolumn{11}{|l|}{70} \\
\hline \multicolumn{11}{|l|}{60} \\
\hline \multicolumn{11}{|l|}{50} \\
\hline \multicolumn{11}{|l|}{40} \\
\hline \multicolumn{11}{|l|}{30} \\
\hline \multicolumn{11}{|l|}{20} \\
\hline CI (U.A.) & & $0( \pm$ & & & , & 67, & & & $( \pm 21$ & \\
\hline
\end{tabular}

FIRURA 2 - Periodização do treinamento para o GE.

\begin{tabular}{|c|c|c|c|c|c|c|c|c|c|c|}
\hline \multirow{2}{*}{$\begin{array}{l}\text { Mesociclo } \\
\text { Microciclo } \\
\end{array}$} & \multicolumn{3}{|c|}{ Preparatório I } & \multicolumn{4}{|c|}{ Preparatório II } & \multicolumn{3}{|c|}{ Polimento } \\
\hline & 1 & 2 & 3 & 4 & 5 & 6 & 7 & 8 & 9 & 10 \\
\hline \multicolumn{11}{|l|}{ Volume (\%) } \\
\hline \multicolumn{11}{|l|}{100} \\
\hline \multicolumn{11}{|l|}{90} \\
\hline \multicolumn{11}{|l|}{80} \\
\hline \multicolumn{11}{|l|}{70} \\
\hline 60 & & & & & & & & & & \\
\hline
\end{tabular}

Continua 
FIGURA 2- Periodização do treinamento para o GE.

\begin{tabular}{|c|c|c|c|c|c|c|c|c|c|c|}
\hline \multirow{2}{*}{\begin{tabular}{|l|} 
Mesociclo \\
Microciclo
\end{tabular}} & \multicolumn{3}{|c|}{ Preparatório I } & \multicolumn{4}{|c|}{ Preparatório II } & \multicolumn{3}{|c|}{ Polimento } \\
\hline & 1 & 2 & 3 & 4 & 5 & 6 & 7 & 8 & 9 & 10 \\
\hline \multicolumn{11}{|c|}{ Volume (\%) } \\
\hline \multicolumn{11}{|l|}{50} \\
\hline \multicolumn{11}{|l|}{40} \\
\hline \multicolumn{11}{|l|}{30} \\
\hline \multicolumn{11}{|l|}{20} \\
\hline CI (U.A.) & \multicolumn{3}{|c|}{$4.615( \pm 226,42)$} & \multicolumn{4}{|c|}{$6,689,18( \pm 312,54)^{a, c}$} & \multicolumn{3}{|c|}{$4.123( \pm 253,38)^{a, b}$} \\
\hline
\end{tabular}

FIGURA 3 - Periodização do treinamento para o GC.

\begin{tabular}{|c|c|c|c|c|c|c|c|c|c|c|}
\hline \multirow{2}{*}{$\begin{array}{l}\text { Mesociclo } \\
\text { Microciclo }\end{array}$} & \multicolumn{3}{|c|}{ Preparatório I } & \multicolumn{7}{|c|}{ Preparatório II } \\
\hline & 1 & 2 & 3 & 4 & 5 & 6 & 7 & 8 & 9 & 10 \\
\hline \multicolumn{11}{|c|}{ Volume (\%) } \\
\hline \multicolumn{11}{|l|}{100} \\
\hline \multicolumn{11}{|l|}{90} \\
\hline \multicolumn{11}{|l|}{80} \\
\hline \multicolumn{11}{|l|}{70} \\
\hline \multicolumn{11}{|l|}{60} \\
\hline \multicolumn{11}{|l|}{50} \\
\hline \multicolumn{11}{|l|}{40} \\
\hline \multicolumn{11}{|l|}{30} \\
\hline \multicolumn{11}{|l|}{20} \\
\hline CI (U.A.) & & 0( & & & 01,3 & 04,6 & & & $9( \pm$ & $12)^{\mathrm{b}}$ \\
\hline
\end{tabular}

\section{Instrumentos}

Utilizou-se a plataforma de força Multisprint Full (Hidrofit ${ }^{\circledR}$, Brasil) para analisar a força explosiva de membros inferiores, conforme metodologia já utilizada em outra investigação ${ }^{3}$. Foram adotadas 3 tentativas para cada atleta com intervalo de 30 segundos entre as tentativas. Computouse o melhor resultado dentre as 3 tentativas. Os atletas realizaram o salto contramovimento, partindo da inércia. Adotou-se o movimento semelhante a realização da técnica de bloqueio. Salienta-se que todos os atletas eram familiarizados com este teste antes do início da investigação.

A maturação biológica foi avaliada por intermédio da maturaçáo somática. Assim, foram aferidos massa corporal, estatura e altura troncocefálica. O comprimento de pernas foi obtido pela diferença entre estatura e altura tronco-cefálica. Estas medidas, juntamente com a idade cronológica foram utilizadas em uma equaçáo estabelecida por Mirwald et al. ${ }^{14}$, que estima a idade do pico de velocidade de crescimento em estatura. Em razáo de achados científicos apontarem influência da maturaçâo biológica sobre variáveis de desempenho físico ${ }^{15}$, decidiu-se controlar (técnicas estatísticas) a idade do pico de velocidade de crescimento em estatura na presente pesquisa.

A densidade corporal foi determinada por meio da técnica de espessura das dobras cutâneas, por meio de um compasso da marca Lange ${ }^{\odot}(\mathrm{USA})$, sendo utilizadas as dobras cutâneas triciptal e subescapular, adotando o protocolo de Slaughter et al. ${ }^{16}$, que leva em consideraçáo a etnia (branca ou negra) e a etapa maturacional em função da idade cronológica (púbere12 a 14 anos; e pós-púbere - 15 a 17 anos) do avaliado. Neste sentido, a etnia foi determinada por meio de autoavaliação. Para as aferiçôes das dobras cutâneas, utilizaram-se as padronizaçôes da Internacional Society for Advancement for Kineanthropometry ${ }^{17}$. O percentual de gordura corporal (\%G) foi determinado por meio da equaçáo de SIRI ${ }^{18}$.

Dados demográficos (idade, etnia, frequência semanal de treino e horas de treino diária) foram avaliados por intermédio de um questionário construído pelos próprios pesquisadores. 


\section{Procedimentos}

A priori, os pesquisadores responsáveis entraram em contato com o diretor das categorias de base de voleibol de um clube do estado de Pernambuco/Brasil. Os procedimentos, bem como os objetivos do estudo foram devidamente explicados e foi solicitada a autorização para desenvolver a investigação com os atletas.

Em seguida, foi realizada uma reunião com os atletas com o intuito de esclarecer sobre todos os procedimentos éticos da investigaçáo. Neste encontro também foi entregue o TCLE ao seu respectivo treinador para o consentimento da participação de seus atletas. Todos os atletas assinaram o termo de assentimento, concordando com a sua participação voluntária na investigação.

As coletas dos dados foram realizadas no local de treinamento (quadra de voleibol). Massa corporal, estatura e dobras cutâneas foram aferidas e em seguida os atletas realizaram o teste de impulsão vertical antes do primeiro microciclo e ao final de cada mesociclo (último microciclo de cada mesociclo). Realizou-se aquecimento (corrida seguida de saltos verticais) com duração de 3 minutos antes da realização do teste de impulsão vertical. Os atletas receberam a mesma orientação para a realização do teste e eventuais dúvidas foram esclarecidas. Foi solicitado aos atletas que não realizassem exercícios físicos nas 24 horas antescedentes ao teste, conforme indicado pela literatura científica ${ }^{19,20}$.

\section{Análise dos dados}

Conduziu-se o teste Shapiro Wilk para avaliar a distribuição dos dados. O teste de Levene foi utilizado para testar a homecedasticidade, ao passo que a esfericidade dos dados foi verificada mediante o teste de Mauchly. Quando esse último pressuposto foi violado, a correção de Greenhouse-Geisser foi adotada. Em razão da não violação paramétrica nos três grupos (GL, GE e GC), optou-se pela utilização de técnicas paramétricas. Conduziu-se a análise univariada de covariância (ANCOVA) de medidas repetidas para comparar a força explosiva de membros inferiores entre os grupos (GL, GE e GC) em função do mesociclo (preparatório I, preparatório II e polimento). O teste post hoc de Bonferroni foi utilizado para identificar a localização das diferenças estatísticas. Salienta-se que a idade do pico de velocidade de crescimento em estatura foi estatisticamente controlada (variável confundidora). Ademais, utilizou-se o tamanho do efeito de Cohen, representado pela sigla " $d$ ”, para revelar diferenças do ponto de vista prático. Foram adotados os seguintes critérios, de acordo com os apontamentos de Thalheimer e $\operatorname{Cook}^{21}: d<0,4=$ baixo tamanho do efeito, $0,4 \leq d>0,8=$ tamanho do efeito moderado e, $d \geq 0,8=$ grande tamanho do efeito. Todos os dados foram tratados no software SPSS 21.0, adotando-se nível de significância de 5\%.

\section{Resultados}

Os dados descritivos [força explosiva de membros inferiores, $\% \mathrm{G}$, idade e regime de treinamento semanal (frequência semanal de treino $\mathrm{x}$ horas de treino diária)] do baseline (pré-teste) podem ser visualizados na TABELA 2.

Em relação a força explosiva de membros inferiores (TABELA 3), os resultados apresentaram efeitos de tempo $\left(F_{(4,38)}=31,93, p=0,01\right) \mathrm{e}$ grupo $\left(F_{(3,39)}=41,87, p=0,01\right)$ que merecem destaque: a) os achados revelaram que a força explosiva de membros inferiores aumentou da pré-temporada até o final da etapa preparatória I no $\operatorname{GL}\left(F_{(2,13)}=27,66, p=0,01\right), \operatorname{GE}\left(F_{(2,12)}=23,07\right.$, $p=0,01)$ e $\mathrm{GC}\left(F_{(2,11)}=18,31, p=0,01\right)$, não sendo identificada diferença significante entre os grupos $\left(F_{(3,39)}=2,70, p=0,17, d=0,1\right)$; b) a ANCOVA náo revelou efeito de tempo $\left(F_{(2,40)}=2,08, p=0,25\right)$ e grupo $\left(F_{(3,39)}=1,62, p=0,28, d=0,1\right)$ para a força explosiva de membros inferiores quando comparadas as etapas preparatória I e II e; c) a força explosiva de membros inferiores aumentou do período preparatório II para o polimento no $\operatorname{GL}\left(F_{(2,13)}=32,55, p=0,01\right)$ e $\operatorname{GE}\left(F_{(2,12)}=29,14\right.$, $p=0,01)$, fato náo revelado para o $\mathrm{GC}\left(F_{(2}\right.$, 11) $=3,48, p=0,19)$. Ademais, foi identificada diferença significante da força explosiva de membros inferiores entre GL e GC $\left(F_{(2,26)}=37,90, p=0,01\right.$, $d=0,7)$, GE e GC $\left(F_{(2,25)}=30,02, p=0,01, d=0,5\right)$ e entre GL e GE $\left(F_{(2,27)}=17,52, p=0,03, d=0,4\right)$ na etapa do polimento, conforme a TABELA 3 indica. Por fim, vale destacar que os resultados da ANCOVA demonstraram relação da idade do pico de velocidade de crescimento em estatura (variável confundidora) com a força explosiva de membros inferiores em todas as etapas da investigaçáo $\left(\mathrm{F}_{(4,38)}=49,33, p=0,001\right)$. 
TABELA 2 - Valores descritivos (média e desvio padrão) das variáveis da pesquisa

\begin{tabular}{lcc}
\hline Variáveis & Média & Desvio padráo \\
\hline Força explosiva de membros inferiores $(\mathrm{cm})$ & 29,30 & 0,05 \\
$\% \mathrm{G}$ & 21,75 & 6,87 \\
Idade (anos) & 15,41 & 1,24 \\
Regime de treinamento semanal (horas) & 10,40 & 0,69 \\
\hline
\end{tabular}

TABELA 3 - Média e erro padrão da força explosiva de membros inferiores [altura (cm)] em razão do grupo (GL, GE e GC) e etapa da periodização (preparatório I, preparatório II e polimento)

\begin{tabular}{lcccc}
\hline Grupo & Pré-temporada & Preparatório I & Preparatório II & Polimento $^{*, \#, \mathbf{\$}}$ \\
\hline GL & $29,33( \pm 0,04)^{\mathrm{d}}$ & $30,14( \pm 0,07)^{\mathrm{a}, \mathrm{d}}$ & $30,17( \pm 0,05)^{\mathrm{a}, \mathrm{d}}$ & $31,26( \pm 0,09)^{\mathrm{b}}$ \\
GE & $29,28( \pm 0,05)^{\mathrm{d}}$ & $30,11( \pm 0,06)^{\mathrm{a}, \mathrm{d}}$ & $30,16( \pm 0,07)^{\mathrm{a}, \mathrm{d}}$ & $30,89( \pm 0,08)^{\mathrm{b}}$ \\
GC & $29,31( \pm 0,05)^{\mathrm{d}}$ & $30,10( \pm 0,04)^{\mathrm{a}}$ & $30,12( \pm 0,08)^{\mathrm{a}}$ & $30,15( \pm 0,05)$ \\
\hline
\end{tabular}

\section{Discussão}

O estudo teve como premissa analisar o efeito do tipo de polimento sobre a força explosiva de membros inferiores em jovens atletas de voleibol do sexo masculino. De maneira geral, os achados revelaram efeitos positivos do polimento sobre a força explosiva de membros inferiores, salientando o maior aumento para o grupo que realizou o polimento linear, o que corrobora as hipóteses formuladas para este estudo.

Os resultados indicaram que a força explosiva de membros inferiores aumentou da pré-temporada até o final da fase preparatória I nos três grupos, sem identificação de diferença entre os grupos. Estudos têm demonstrado que a força explosiva de atletas pode aumentar logo no início do temporada competitiva $^{22,23}$. Este fato pode ser explicado em razão da redução do desempenho físico após o período transitório entre duas temporadas competitivas, também conhecido como efeito destreinamento ${ }^{24}$. Por conseguinte, os atletas retornam a sua rotina de treinamento, o que acarreta aumento progressivo do desempenho físico ${ }^{22}$, por exemplo a força explosiva, o que, de certo modo, pode explicar o aumento desta variável ao final do período preparatório I no presente estudo. De fato, duas a três semanas de treinamento físico (força, resistência aeróbia e velocidade/agilidade) são suficientes para aumentar a força explosiva em atletas ${ }^{23}$, o que corrobora os achados da presente investigação.

Considerando o período preparatório II, a força explosiva de membros inferiores não demonstrou alteração em comparação ao período preparatório I nos três grupos. Segundo MuJıKA ${ }^{9}$, potência anaeróbia, potência aeróbia, velocidade/agilidade e/ou força muscular costumam não melhorar após fases de intensificação do treinamento. Do mesmo modo, Le Meur et al. ${ }^{6}$ ressaltam que o desempenho físico pode não alterar após períodos de aumento das cargas de treinamento, corroborando os achados da presente pesquisa. Embora os atletas suportem cargas maiores de treinamento durante a fase preparatória II, vale destacar que repetidas sessóes de alta intensidade geram aumento de danos musculares, o que pode acarretar reduçáo e/ou manutenção do desempenho físico ${ }^{2}$.

A respeito da etapa do polimento, os grupos experimentais (GL e GE) aumentaram a força explosiva de membros inferiores ao final desta fase, fato não identificado para o GC. Parece que a manutenção da intensidade conjugada com a redução do volume das sessóes de treino durante duas a quatro semanas pode potencializar o desempenho físico de atletas ${ }^{11}$, explicando assim, os achados para o GL e GE. Todavia, a literatura científica ainda não entrou em consenso concernente a melhor estratégia de prescrição do polimento para atletas de esportes intermitentes. Por um lado, pesquisadores indicam o polimento linear ${ }^{10,25}$. Por outro, recomenda-se adotar o polimento por etapa ${ }^{6}$. Considerando os resultados da presente investigação, ambas as estratégias de polimento foram eficazes para otimizar a força explosiva de membros inferiores, embora o GL tenha melhorado mais do que o GE, sendo revelado moderado tamanho do
\%G: percentual de gordura corporal.

\footnotetext{
GL: periodização linear; GE: periodização por etapa; GC: grupo controle; ${ }^{\text {a }} \mathrm{p}<0,05$ em relação a "Pré-Temporada"; b $p<0,05$ em relação a "Preparatório I"; ${ }^{\text {d }}$ $p<0,05$ em relação a "Polimento"; " $p<0,05$ diferença entre $\mathrm{GL}$ e GE; $\# p<0,05$ diferença entre $\mathrm{GL}$ e GC; ${ }^{\$} p<0,05$ diferença entre GE e GC.
} 
efeito (comparação dos valores pontuais), o que indica razoável probabilidade deste fato ser verdadeiro para jovens atletas com características semelhantes a do presente estudo. Cabe salientar, sobretudo, que a manutençáo de cargas elevadas de treinamento pode náo ser uma estratégia adequada de periodizaçáo, fato revelado para o $\mathrm{GC}$, pois estes atletas não potencializaram a força explosiva de membros inferiores após a etapa preparatória II.

Concernente ao pico de velocidade de crescimento em estatura, os achados indicaram relação com a força explosiva de membros inferiores em todas as etapas da investigação. De fato, a maturação biológica exerce influência em parâmetros de desempenho físico $^{15}$. Logo, atletas mais avançados biologicamente normalmente demonstram melhor desempenho em habilidades que exigem força e velocidade.

O presente estudo, apesar de revelar resultados interessantes, é dotado de limitaçóes que devem ser mencionadas. A alimentação dos atletas não foi controlada, o que pode ter afetado os resultados do estudo. Aponta-se também o uso de método duplamente indireto (dobras cutâneas) para se avaliar o percentual de gordura como limitação. Assim, os achados devem ser tratados com cautela.

Baseado nos achados da pesquisa, concluiu-se que as estratégias de polimento linear e por etapa foram eficientes para otimizar a força explosiva de membros inferiores de jovens atletas de voleibol. No entanto, vale ressaltar que a estratégia de polimento linear revelou maior melhora na força explosiva de membros inferiores quando comparada a estratégia de polimento por etapa. Segundo alguns pesquisadores ${ }^{7-9}$, o desempenho físico de atletas após se reduzir repentinamente o volume de treinamento (como é adotado no polimento por etapa) costuma aumentar no início desta fase, mais especificamente, nas duas primeiras semanas. Parece que alguns atletas não conseguem manter o nível de desempenho físico a partir da terceira semana de polimento por etapa ${ }^{10}$. Além disso, investigaçóes revelaram que os níveis de adaptaçóes neurais (tamanho e velocidade de recrutamento das fibras musculares) e metabólicas (estoques musculares de glicogênio) oriundas do polimento por etapa reduziram após a segunda semana de redução repentina do volume, o que pode explicar a diferença encontrada entre o GE e GL após as três semanas de polimento. Por outro lado, de acordo com Pyne et al. ${ }^{11}$, a atenuação gradual do volume de treinamento (como é adotado no polimento linear) é um método mais efetivo para otimizar o desempenho físico de atletas após três a quatro semanas. Pesquisas demonstraram que a redução linear do volume de treinamento gera vagarosamente atenuação do acúmulo de estresse psicofisiológico no atleta, revelando baixos níveis de fadiga sem que haja perda das adaptaçóes fisiológicas após aproximadamente três semanas de polimento ${ }^{6,7}$. Por fim, concluiu-se que a manutenção de elevadas cargas de treinamento pode não ser eficiente para potencializar a força explosiva de membros inferiores no voleibol. Segundo Nogueira et al. ${ }^{1}$, a manutenção das cargas de treinamento pode acarretar aumento da monotonia de treino, o que por sua vez, parece ter relação com a estagnação ou diminuiçáo do desempenho dos atletas².

\section{Abstract}

\section{Can lower limbs explosive strength be modified by type of tapering in volleyball athletes?}

The aim was to analyze the effect of the type of tapering on lower limbs explosive power (LEP) in young volleyball athletes. 42 athletes aged between 15 and 17 years were randomly divided into 3 groups: linear $(\mathrm{LG})$, by stage $(\mathrm{SG})$ and control (CG). All groups did the same planning training to the tapering phase. Only the CG did not perform tapering. Tapering lasted three weeks, adopting the linear tapering method for LG and tapering by step for SG. We used the force platform to assess the LEP. This instrument was used prior to the start of the season and the last week of each mesocycle. It led to univariate analysis of covariance (ANCOVA) for repeated measures to compare the LEP between groups according to mesocycle. The results presented time $\left(F_{(4,38)}=31.93, p=0.01\right)$ and group $\left(F_{(3,39)}=41.87, p=0.01\right)$ effect. The LEP increased in LG $\left(F_{(2,13)}=32.55, p=0.01\right)$ and SG $\left(F_{(2,12)}=29.14, p=0.01\right)$ after tapering, fact undisclosed to $\operatorname{CG}\left(F_{(2,11)}=3.48\right.$, $p=0.19)$. Overall, the findings of this study revealed increase of LEP after tapering to LG and SG. It was concluded that the tapering was effective in improving the LEP in volleyball athletes.

KEYWORDS: Strength; Performance; Athletes. 


\section{Agradecimentos}

Aos atletas pela colaboração em participar da investigação. Ao Banco Santander pelo financiamento de parte do projeto.

\section{Referências}

1. Nogueira FCA, Nogueira RA, Coimbra DR, Miloski B, Freitas VH, Bara-Filho MG. Internal training load: perception of volleyball coaches and athletes. Rev Bras Cineantropom Desempenho Hum. 2014;16(6):638-47.

2. Freitas VH, Miloski B, Bara-Filho MG. Monitoramento da carga interna de um período de treinamento em jogadores de voleibol. Rev Bras Educ Fís Esporte. 2015;29(1):5-12.

3. Alves CVV, Santos LR, Vianna JM, Novaes GS, Damasceno VO. Força explosiva em distintos estágios de maturaçáo em jovens futebolistas das categorias infantil e juvenil. Rev Bras Ciênc Esporte. 2015;37(2):199-203.

4. Seiler $\mathrm{S}$. What is best practice for training intensity and duration distribution in endurance athletes? Int J Sports Physiol Perform. 2010;5(3):276-91.

5. Hartmann H, Wirth K, Keiner M, Mickel C, Sander A, Szilvas E. Short-term periodization models: Effects on strength and speed-strength performance. Sports Med. 2015;45(10):1373-86.

6. Le Meur Y, Hausswirth C, Mujika I. Tapering for competition: a review. Sci Sports. 2012;27:77-87.

7. Mujika I, Chaouachi A, Chamari K. Precompetition taper and nutritional strategies: special reference to training during Ramadan intermittent fast. Br J Sports Med. 2010;44(7):495-501.

8. Bosquet L, Montpetit J, Arvisais D, Mujika I. Effects of tapering on performance: a meta-analysis. Med Sci Sports Exercise. 2007;31(8):1358-65.

9. Mujika I. Intense training: the key to optimal performance before and during the taper. Scand J Med Sci Sports. 2010;20 (suppl 2):24-31.

10. Hellard P, Avalos M, Hausswirth C, Pyne D, Toussaint J, Mujika I. Identifying optimal overload and taper in elite swimmers over time. J Sports Sci Med. 2013;12(4):668-78.

11. Pyne DB, Mujika I, Reilly T. Peaking for optimal performance: Research limitations and future directions. J Sports Sci. 2009;27(3):195-202.

12. Ronnestad BR, Ellefsen HS. Block periodization of high-intensity aerobic intervals provides superior training effects in trained cyclists. Scan J of Med Sci Sports. 2014;24(1):34-42.

13. Foster C, Florhaug JA, Franklin J, et al. A new approach to monitoring exercise training. J Strength Cond Res. 2001;15(1):109-15.

14. Mirwald RL, Baxter-Jones ADG, Bailey DA, Beunen GP. An assessment of maturity from anthropometric measurements. Med Sci Sport Exerc. 2002;34(4):689-94.

15. Matta MO, Figueiredo JFB, Garcia ES, Seabra AFT. Morphological, maturational, functional and technical profile of young Brazilian soccer players. Rev Bras Cineantropom Desempenho Hum. 2014;16(3):277-86.

16. Slaughter MH, Lohman TG, Boileau R, Hoswill CA, Stillman RJ, Yanloan MD. Skinfold equations for estimation of body fatness in children and youth. Hum Biol. 1988;60(5):709-23.

17. The Internacional Society for Advancement for Kineanthropometry [Internet]. Canberra: National Library of Australia; 2013 [citado 12 jul 2013]. Disponível em: http://www.isakonline.com.

18. Siri WE. The gross composition of the body. In: Tobias CA, Lawrence JH, editors. Advances in biological and medical physics. New York: Academic Press; 1956. p. 239-80.

19. Loturco I, Nakamura FY, Tricoli V, et al. Determining the optimum power load in jump squat using the mean propulsive velocity. PloS One. 2015. In press.

20. Loturco I, Kobal R, Gil S, et al. Differences in loaded and unloaded vertical jumping ability and sprinting performance between Brazilian elite under-20 and senior soccer players. American J Sports Sci. 2014;2(6-1):8-13.

21. Thalheimer W, Cook S. How to calculate effect sizes from published research articles: a simplified methodology [Internet]. 2009 [citado 26 jul 2019]. Disponível em: https://www.researchgate.net/publication/253642160_How_to_calculate_effect_ sizes_from_published_research_A_simplified_methodology.

22. Loturco I, Nakamura FY, Kobal R, et al. Training for power and speed: effects of increasing or decreasing jump squat velocity in elite Young soccer players. J Strength Cond Res. 2015;29(10):2771-9. 
Fortes LS, et al.

23. Ramirez-Campillo R, Vergara-Pedreros M, Henriquez-Olguin C, et al. Effects of plyometric training on maximal-intensity exercise and endurance in male and female soccer players. J Sports Sci. 2015. In press.

24. Bosquet L, Berryman N, Dupuy O, et al. Effect of training cessation on muscular performance: a meta-analysis. Scand J Med Sci Sports. 2013;23(3):e140-9.

25. Mujika I, Padilla S, Pyne D. Swimming performance changes during the final 3 weeks of training leading to the sydney 2000 olympic games. Int J Sports Med. 2002;23(8):582-7.

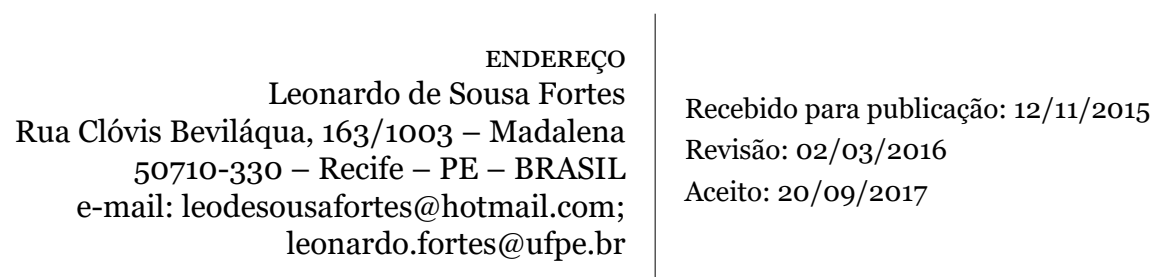

